Published in final edited form as:

Curr Hematol Malig Rep. 2014 June ; 9(2): 193-201. doi:10.1007/s11899-014-0205-6.

\title{
Contemporary Treatment of APL
}

\author{
Elizabeth H. Cull, MD and Jessica K. Altman, MD
}

Division of Hematology and Oncology, Department of Medicine, Northwestern University

Feinberg School of Medicine and Northwestern Memorial Hospital, Chicago, IL, USA

\begin{abstract}
Acute promyelocytic leukemia (APL) is characterized by coagulopathy, leukopenic presentation and sensitivity to anthracyclines, all-trans retinoic acid (ATRA) and arsenic trioxide (ATO). For the last 25 years, APL has been treated with a combination of ATRA and chemotherapy for induction followed by consolidation and maintenance therapy. This general treatment approach has resulted in cure rates of 80-90\%. ATO, originally approved in relapsed APL, has been incorporated into contemporary upfront treatment regimens with excellent response rates. Recent studies show that most patients with APL can be cured with ATRA and ATO alone, eliminating cytotoxic chemotherapy and resulting in superior outcomes compared to standard treatment. We will herein review historical treatment of APL, treatment considerations in specific patient populations, and therapeutic updates.
\end{abstract}

\section{Keywords}

acute promyelocytic leukemia; arsenic trioxide; all-trans retinoic acid; early death rate

\section{Introduction}

Acute promyelocytic leukemia (APL) is a subtype of acute myeloid leukemia (AML) characterized by leukopenia, coagulopathy and high cure rate. APL is a rare disease with approximately 1000 cases diagnosed yearly in the United States [1]. Patients typically present with pancytopenia and complications from coagulopathy $[1,2]$. Over $95 \%$ of cases are characterized by a balanced translocation of the PML gene on chromosome 15 and the RARA gene on chromosome 17, $t(15 ; 17)$. This results in a PML/RARA fusion protein that inhibits transcription of genes necessary for differentiation. APL cells are sensitive to the differentiating effects of all-trans retinoic acid (ATRA) and arsenic trioxide (ATO) [3]. Differentiation of leukemic promyelocytes leads to rapid resolution of coagulopathy, the major cause of early death in APL patients. Studies with single agent ATRA began in the 1980s [4-10], and since that time, ATRA and ATO in varying combinations with chemotherapeutic agents have resulted in cure rates of $80-90 \%$ [11]. Based on recent phase

Address Correspondence to: Elizabeth H. Cull, MD, Northwestern University Feinberg School of Medicine, Division of Hematology/ Oncology, Robert H. Lurie Comprehensive Cancer Center Northwestern University, 676 N. St. Clair, Suite 850, Chicago, IL 60611, Phone number: 312-695-0990, Fax number: 312-695-6189, e-cull@northwestern.edu.

Jessica K. Altman, MD, Northwestern University Feinberg School of Medicine, Division of Hematology/Oncology, Robert H. Lurie Comprehensive Cancer Center Northwestern University, Lurie 5-111, 303 E. Superior Street, Chicago, IL 60611, Phone number:

312-503-1817, Fax number: 312-503-0189, j-altman@ northwestern.edu 
III data presented by the Italian Group for Haematological Diseases in Adults (GIMEMA), there is now evidence to suggest that chemotherapy can be eliminated completely for low and intermediate risk patients [12]. In this article, we will review the historical data informing current APL treatment and discuss clinical considerations in specific patient populations and recent therapeutic updates.

\section{Historical Overview}

With efficacy first reported in the 1980s by investigators from the People's Republic of China, single agent ATRA has been found to induce high complete remission (CR) rates in APL [4-10]. In the 1990s, European and American investigators added ATRA to chemotherapy further improving survival rates [13-16]. The focus then shifted to riskadapted strategies for APL treatment, partitioning patients into low, intermediate and high risk groups based on white blood cell (WBC) count and platelet count [17-20]. Specifically, WBC over $10,000 / \mu \mathrm{L}$ was found to correlate with increased induction death and higher relapse rates [13-16].

ATO was also found to be highly active in APL. ATO breaks down the PML-RAR- $\propto$ fusion transcript, allowing for transcription of genes necessary for differentiation of promyelocytic leukemia cells and apoptosis [21]. ATO was initially approved for use in the relapsed setting $[22,23]$. Studies then transitioned to its use in the upfront setting in combination with chemotherapy and ATRA resulting in impressive outcomes [24-28]. These studies led to the pivotal phase III randomized trial (APL0406) in which ATRA/ATO was shown to be noninferior to ATRA/chemotherapy for low/intermediate risk patients [12]. Tables 1 and 2 summarize the regimens and outcomes data from significant APL trials conducted in the last 20 years.

\section{Treatment considerations in standard risk APL}

\section{Eliminating chemotherapy from treatment}

Treatment for low and intermediate risk APL, referred to here as standard risk APL has been redefined based on results from the APL0406 trial. Shen initially reported the benefits of ATRA in addition to ATO in 2004. 61 newly diagnosed patients with APL were randomized to ATRA vs. ATO vs. ATRA/ATO, and CR rates of over $90 \%$ were achieved in all arms; however, the combination arm had an improved relapse free survival (RFS) and shorter time to hematologic remission [28]. Estey confirmed a benefit for ATRA plus ATO, achieving a CR rate of 95\% in low-risk patients and 79\% in high-risk patients [24, 27]. The North American Leukemia Intergroup Study C9710 also evaluated adding ATO to consolidation with ATRA/chemotherapy and reported improved outcomes in the groups receiving ATO [26]. The APML4 study added ATO to ATRA and anthracycline induction and eliminated chemotherapy from consolidation, utilizing 2 cycles of ATRA/ATO followed by 2 years of maintenance therapy. Investigators reported a $95 \%$ hematologic CR following induction and a 2-year OS of $94 \%$ [25]. An update presented at the 2013 Rome APL meeting reported a sustained 5-year OS of 94\% [29]. The APL0406 trial built on data obtained from these previous studies. In this study, newly diagnosed patients with standard risk APL were randomized to ATRA/ATO for induction and consolidation therapy or induction therapy 
with ATRA/idarubicin followed by ATRA/chemotherapy consolidation and 2 years of maintenance therapy. At 34.4-month follow-up, EFS was 97\% in the ATRA/ATO group and $86 \%$ in the ATRA/chemotherapy group. Additionally, the ATRA/ATO group had improved OS compared to the ATRA/chemotherapy group (99\% vs. 91\%). This improvement in OS was mostly due to reduced treatment related toxicity rather than death from relapse. Specifically, rates of neutropenia and infection were higher in the ATRA/chemotherapy group; the incidence of hepatotoxicity and QTc prolongation were increased in the ATRA/ATO group, but in the majority of cases, these findings were reversible with ATO withdrawal. Therefore, not only was toxicity improved with ATRA/ATO, relapse rates were similar between groups as well implying that disease free survival was not compromised by less intensive therapy [12]. Additionally, with elimination of anthracyclines from the regimen, long-term side effects including cardiac toxicity and secondary myeloid neoplasms may be reduced. Based on this study, ATRA/ATO is now a recommended upfront regimen for standard risk APL while previously it was recommended in the relapsed setting or in patients unable to receive anthracyclines [30]. Notably, the role of ATO based induction in high-risk APL or in patients over age 70 was not addressed by this trial.

\section{Consolidation Therapy}

Consolidation regimens in an anthracycline based induction approach vary and recent studies have focused on a risk-adapted strategy when choosing consolidation agents. The LPA96 and LPA99 studies used the same AIDA (ATRA/idarubicin) induction regimen but varied the consolidation regimen based on risk of relapse $[15,18,19]$. The LPA 99 study specifically confirmed the benefit of ATRA use in consolidation (in addition to intensified chemotherapy), most notably in the intermediate and high-risk groups. The French APL 2000 study and the LPA 2005 study further defined the role of cytarabine in consolidation therapy [20,31]. A comparison of the LPA 99 trial and the French APL 2000 study showed that cytarabine given in induction/consolidation was beneficial in the high-risk group but did not benefit the standard-risk group [17].

More recently, the question of ATO use in consolidation has been explored. The APML4 study used ATO/ATRA/idarubicin in induction followed by 2 cycles of ATRA/ATO in consolidation in all risk patients and reported a 2-year OS of 93.2\% [25]. The North American Intergroup trial C9710 confirmed a benefit of ATO use in consolidation in all patients [26]. The APL0406 study also used ATO/ATRA for consolidation in the study arm and reported EFS rates and OS rates superior to the standard chemotherapy arm [12]. In summary, several consolidation regimens are appropriate, and a risk-adapted strategy should be used when selecting therapy in APL. However, it should be emphasized that regimens should generally not be mixed and matched but rather followed in entirety per protocol from induction through maintenance. This allows the possibility of optimal results as demonstrated in the corresponding trials.

\section{Role of maintenance}

In contrast to other subtypes of AML, consolidation has traditionally been followed by two years of maintenance therapy with ATRA, 6-mercaptopurine and methotrexate [13]. Now that ATO is being used in induction and/or consolidation, need for maintenance has been 
questioned. In the phase II study published by Estey, low risk patients received ATRA/ATO for induction followed by intermittent ATRA/ATO for a total of 28 weeks after CR was confirmed. High-risk patients also received gemtuzumab ozogamicin (GO). Maintenance therapy was eliminated $[24,27]$. At a median follow-up of 99 weeks, OS was $85 \%$. Not only did this study suggest that chemotherapy may not be required to achieve cure in APL, it also questioned the role of maintenance therapy. A phase III SWOG study sought to examine the role of maintenance therapy. In this trial, standard-risk patients received a standard induction and consolidation regimen including ATO and were then randomized (if in molecular remission) to one year of maintenance therapy vs. observation. Although the study was closed due to slow accrual, at 22.7-month follow-up, the OS in all patients was $>93 \%$ and no relapses were noted in either group [32]. The APL 0406 study of ATRA/ATO for induction confirmed that survival and relapse rates are not compromised by deletion of maintenance therapy when ATO is used in induction and consolidation. However, median follow-up was only 34.4 months and longer follow-up may be needed in all of these studies to ensure that maintenance therapy can indeed be eliminated [12].

\section{Complications and management of high risk APL}

\section{Increased Early Death Rate}

$20-25 \%$ of APL patients present with $\mathrm{WBC}$ over $10,000 / \mu \mathrm{L}$, and these high-risk patients have both a higher early death rate (EDR) and an increased risk of relapse. They typically present with more severe coagulopathy and have higher rates of life threatening bleeding [33]. Even in the post-ATRA era, EDR has been reported between 5-20\% in this subgroup of patients $[13,14,19,27,33]$. This rate may even be an underestimate as it only encompasses patients treated at academic centers and/or stable enough for clinical trial enrollment. One single center study reviewed data on their APL patients not enrolled on clinical trials and found an EDR of $21 \%$ compared to $3 \%$ in patients enrolled on clinical trials [34]. Patients not enrolled tended to have higher WBCs and lower platelet counts. Despite major advances in APL therapies, the EDR remains high, mostly due to hemorrhagic complications. In the Swedish APL registry, reported EDR was 29\% and death occurred more commonly in elderly patients and patients with poor performance status. The majority of early deaths were due to bleeding, followed by cardiopulmonary failure and sepsis [35]. In a retrospective study of APL patients treated at 5 centers, including our institution, the death rate in the first 30 days was $11 \%$ and $61 \%$ of these deaths were attributable to hemorrhage. There was an increase in hemorrhage following delay of ATRA administration, resulting in increased EDR only in the high risk group [36]. Other studies suggest that early ATRA may not necessarily decrease the EDR however [37]. Despite this, ATRA should still be administered at first suspicion of APL [38]. The importance of aggressive transfusion support is also important. We suggest maintaining a platelet count greater than 30,000-50,000/ $\mathrm{LL}$ and fibrinogen level more than $150 \mathrm{mg} / \mathrm{dL}$ [39]. Although ATRA does reverse the coagulopathy generally in a few days, aggressive blood product support is necessary in the days following suspicion and diagnosis of APL to decrease the EDR [40]. 
Additionally, the risk of developing APL differentiation syndrome (DS) increases with rising WBC count and is another cause of early death [41]. DS is characterized by fevers and respiratory distress as well as interstitial pulmonary infiltrates, pleural effusions, weight gain and lower extremity edema [42]. This syndrome is often mistaken for pneumonia, volume overload or alveolar hemorrhage. Prophylaxis with high dose dexamethasone was used in the APL 2000 trial and led to a decrease in DS related deaths to 3.9\% compared to 5.7\% reported in the earlier APL 93 trial [43]. If not used preemptively, steroids should be started at the earliest sign of DS. If symptoms are life threatening, ATRA or ATO should be temporarily discontinued until symptoms resolve. Prophylactic steroid use is recommended in high-risk patients and should be strongly considered in patients treated with ATRA/ATO for induction as was used preemptively in patients treated with ATRA/ATO on the GIMEMA APL 0406 trial [30].

While most patients with APL present with lower WBCs compared to other AML subtypes, $20-25 \%$ will present with a WBC over $10,000 / \mu \mathrm{L}$ and $5 \%$ or fewer patients will present with a WBC greater than 50,000/ $\mu \mathrm{L}$ [41]. Leukapheresis is generally not recommended in APL given early reports of death following this procedure [44]. Additionally, placement of a central venous catheter necessary for leukapheresis is not recommended given the underlying coagulopathy. Cytotoxic chemotherapy in combination with ATRA should begin promptly in this high-risk group. Hydroxyurea can also be used as a temporizing measure to control leukocytosis [12].

\section{Increased Relapse Rates}

Relapse rates are also greater in the high-risk group. Several studies have shown cytarabinecontaining regimens used during induction or consolidation lead to higher OS and lower relapse rates in high-risk APL [17,31]. Additionally, maintenance therapy is recommended in the high-risk group. The APL 93 trial showed a clear benefit for combined maintenance therapy, most noticeable in high-risk patients [13]. A comparison of the APL 93 trial and APL 2000 trial showed that systematic maintenance therapy as received in the APL 2000 trial was the strongest factor associated with improved OS in the high-risk group [43].

The role of ATO is less well-defined and is not routinely recommended for induction therapy in high-risk groups, apart from in the APML4 regimen. Its use in consolidation is better studied. The North American Intergroup trial C9710 randomized patients to consolidation with ATO following standard daunorubicin/ATRA/cytarabine induction and showed a benefit in all risk groups receiving ATO. Notably, DFS was similar between the standard risk group that did not receive ATO and the high-risk group that received ATO suggesting that addition of ATO may partially overcome the negative prognosis associated with high-risk disease [26].

In regards to CNS relapse, the cumulative rate of CNS relapse in high-risk APL patients is 5\% [45]. Outcomes in patients with CNS relapse are poor and given the increased incidence in high-risk APL, some authors are proponents for prophylactic intrathecal therapy; however, others do not consider this a standard recommendation, given that the number to treat to have a benefit is quite large [38, 45-47]. Furthermore, cytarabine and ATO both cross the blood-brain barrier and may obviate the need for prophylactic intrathecal therapy. 


\section{Considerations in elderly patients with APL}

APL is relatively uncommon in patients over age 60 , representing only $15-20 \%$ of patients diagnosed [48]. The EDR is higher in this cohort due to greater comorbidities compared to their younger counterpart. In a Swedish Leukemia registry study, the EDR was 29\% in all age groups but 50\% in patients over age 60 [35]. Similarly, a study using the Surveillance, Epidemiology, and End Results (SEER) data from the United States reported an EDR of $24 \%$ in APL patients over age 55 [49]. Although EDR and treatment related mortality are higher in this subgroup, patients tend to present with lower risk disease and have lower rates of relapse. The European APL group reviewed outcomes in elderly patients from the APL 93 trial and reported a 4-year OS of 57.8\% in elderly patients compared to $78 \%$ in younger patients. Elderly patients achieved lower rates of CR ( $86 \%$ vs. $94 \%)$ and more died in CR (18.6\% vs. $5.7 \%)$, mostly as a result of complications during consolidation and maintenance therapy. However, 4-year relapse rates were lower in the elderly group compared to the younger cohort (15.6\% vs. 23.2\%) [50]. Results from other studies report similar findings in their elderly cohorts: lower CR rates, higher death rates while in CR, mostly as complications from myelosuppression, resulting in worse OS [51, 52]. In an attempt to decrease these treatment related deaths, the GIMEMA group amended their AIDA protocol to decrease the consolidation courses in elderly patients from three cycles to one [53]. This resulted in an improved OS in the amended protocol cohort compared to the original cohort due to a decrease in treatment related death.

ATO has a more favorable side effect profile compared to conventional chemotherapy and is an attractive option in this elderly cohort that is particularly susceptible to treatment related toxicities. Most studies that use ATO +/- ATRA in the upfront or relapsed setting do not report specifically on outcomes in the elderly. The Chinese group used single agent ATO for remission induction and post-remission therapy for up to 4 years in patients over age 60 . $88 \%$ achieved CR and 10-year rate of relapse, OS and cause-specific survival were 10.3\%, $69.3 \%$ and $84.8 \%$, respectively. Post-remission therapy with ATO was well tolerated, and no ATO-related deaths occurred. The main side effect from therapy was leukocytosis with resulting DS which was successfully managed in all patients. On ten-year follow up, there was no increased rate of malignancy or arsenic poisoning identified [54].

GO has also been studied in elderly patients and shown to be efficacious $[27,55,56]$; however, its approval was withdrawn from the US market and the ability to utilize GO is severely limited. The APL 0406 trial showing superiority of ATRA/ATO over ATRA/ chemotherapy is particularly relevant for elderly APL patients; however, the upper limit of age enrollment was 71 years old. The original publication did not stratify patients by age but the authors recently submitted a letter addressing outcomes in patients ages 60-70. They report similar findings seen in the younger cohort including improved EFS and improved toxicity profile in the ATRA/ATO arm. These findings were non-significant but the numbers analyzed were small [57]. Given the low toxicity of ATRA/ATO, this regimen may be especially appropriate for the elderly population but further studies are required. 


\section{Considerations in relapsed APL}

With current treatment regimens, approximately $10 \%$ of APL patients will relapse [46].

Most studies use ATO alone or in combination with other agents in the relapsed setting [22, 23, 58-62]. Soignet reported results of 40 patients with relapsed APL. Patients received ATO for induction followed by consolidation and maintenance with ATO. $85 \%$ of patients achieved CR; at 18 months follow-up, the OS was 66\% and relapse free survival was 56\% [58]. Shigeno used single agent ATO for induction and consolidation for 34 patients with relapsed APL; they reported a 2-year OS of 56\% [61]. One small trial by French investigators randomized relapsed patients to ATRA/ATO or ATO alone [60]. Outcomes were similar between groups leading its authors to conclude that there was no benefit to adding ATRA to ATO. Current guidelines suggest ATO +/- ATRA for treatment of relapsed disease; however, now that ATRA/ATO is recommended first line, it is unclear how this will affect management of relapsed disease [30]. A retrospective report was recently published of 25 patients with relapsed APL who had been exposed to upfront ATRA/ATO in addition to cytotoxic agents. Patients received a variety of reinduction regimens at relapse including ATRA/ATO or chemotherapy regimens. The authors reported a 40\% CR rate in the patients receiving ATRA/ATO; however, complete molecular remission was low and relapse remained high [63]. For patients with relapsed APL after prior ATRA/ATO exposure, a trial of re-induction with ATRA/ATO is appropriate if sufficient time has passed from last ATO exposure (6-12 months). Alternatively, standard ATRA/chemotherapy regimens can be utilized. Once CR is obtained, we would recommend following guidelines for consolidation with an autologous or allogeneic stem cell transplant (SCT) depending on molecular status.

After attaining a second remission, CSF evaluation and intrathecal chemotherapy is recommended given the risk of CNS involvement in relapsed APL. Autologous SCT is also recommended as post-remission therapy in patients in complete molecular remission (CMR). In a retrospective study of 37 patients with relapsed APL, patients received induction and consolidation with ATO alone or ATO in combination with other agents. Thirty-three patients (89\%) achieved a second remission and of these, 14 received an autologous SCT and 19 received monthly ATO +/- ATRA for 6 months. At a median 32month follow-up, EFS was $83 \%$ in the transplant arm compared to $34.4 \%$ in ATO +/ATRA arm [64]. Autologous SCT has been compared to allogeneic SCT in the relapsed setting as well. In a retrospective study by French investigators, 122 patients with relapsed APL in second remission received autologous SCT or allogeneic SCT. Seven-year RFS and OS were $79.4 \%$ and $59.8 \%$ in the autologous SCT group compared to $92.3 \%$ RFS and $51.8 \%$ OS in the allogeneic SCT group. OS was significantly lower in the allogeneic SCT group secondary to increased treatment related toxicity [65]. Investigators from MD Anderson retrospectively reviewed outcomes in 40 relapsed APL patients undergoing autologous SCT, allogeneic SCT or chemotherapy alone. They found no difference in EFS or OS between groups but the numbers were small and there was a trend toward improved outcomes in the autologous SCT group [66]. Therefore, autologous SCT in second CMR is recommended in patients able to receive the high dose chemotherapy required for conditioning. Allogeneic SCT should be reserved for patients that do not achieve CMR. In 
this group, the graft vs. leukemia effect leads to a decreased rate of relapse. If patients are not transplant eligible, consolidation with ATO or enrollment on a clinical trial is recommended [30].

\section{New Drugs and Formulations}

\section{Oral Arsenic}

ATO has only been available in IV forms until recently. There is considerable interest in developing an oral formulation of ATO. This would have particular relevance in the long consolidation phase in which patients must come to the office daily for IV ATO. A recent phase III randomized trial compared IV ATO to a new oral formulation of ATO and demonstrated non-inferiority of oral ATO to IV ATO, showing comparable rates of CR, OS and adverse events [67]. This oral form of ATO is not currently available in the United States, but there is interest among investigators throughout North America, Europe, and Australia in developing an oral formulation of ATO.

\section{Liposomal ATRA}

Lipo-ATRA, a liposomal form of ATRA, was initially studied with the intent of trying to eliminate chemotherapy in APL treatment. In a study with healthy volunteers, plasma levels of lipo-ATRA were 15-fold elevated compared to oral ATRA and the half-life was also prolonged [68]. Initiated in 1997 prior to the advent of ATO, a prospective trial was performed using lipo-ATRA alone in 34 patients with untreated APL and results were compared to historical controls who had received the AIDA regimen. The CR rate in the standard risk group was $92 \%$, similar to CR rates achieved in historical controls; however, in the high-risk group, the $\mathrm{CR}$ rate was only $38 \%$, significantly lower than matched historical controls [69]. At 13-year follow up, the authors reported an OS of 65\% and no secondary malignancies noted [70]. Lipo-ATRA is not routinely used or readily available but could be considered in situations where oral ATRA is not feasible.

\section{Tamibarotene}

Tamibarotene is a synthetic retinoid, developed and approved in Japan for treatment of relapsed/refractory APL prior to widespread use of ATO in the relapsed setting. ATRA resistance is uncommon but when it does occur, is postulated to be secondary to RAR-a mutations, increased levels of cellular retinoic acid binding protein (CRABP), accelerated elimination of ATRA and other genetic mutations [71, 72]. Tamibarotene exhibits more potent differentiation ability compared to ATRA and is also more specific in its binding. Tamibaratone only binds to RAR- $\alpha$ and RAR- $\beta$ while ATRA also binds to RAR- $\gamma$ (present on dermal epithelium). This difference in binding suggests that tamibarotene may be associated with fewer rashes and other skin toxicities than ATRA. Additionally, tamibarotene, unlike ATRA, does not have a high binding affinity to CRABP, suggesting it may be more active in an ATRA-resistant setting. [73]. One small Japanese study was performed in which 24 evaluable patients with relapsed APL following ATRA exposure received tamibarotene. 58\% of patients achieved a CR. The most significant serious side effect was hyperlipidemia but overall, treatment was well tolerated [74]. Data with this drug is limited in the US and Europe, used in the relapsed/refractory setting [75, 76]. A phase II 
clinical trial (STAR-1), evaluating tamibarotene in the relapsed setting following prior ATRA and ATO therapy, was initiated with results currently pending.

\section{Conclusions}

Treatment for APL is one of the major success stories in oncology. APL was once the most deadly form of AML and is now the most treatable. Cure rates have improved significantly after ATRA was added to chemotherapy, and with the advent of ATO in combination with ATRA, it is now possible to cure many patients without chemotherapy. Given that APL more commonly occurs in younger patients, decreasing secondary malignancies by avoidance of chemotherapy is of paramount importance. Furthermore, utilization of ATO/ ATRA decreases treatment related toxicity and may be particularly beneficial for older adults. EDR continues to be a pressing problem, even at experienced centers, and maintaining a high suspicion of APL, employing aggressive blood product support, and initiating ATRA early should be utilized to minimize early deaths. Further study is required to determine therapeutic options for recurrent disease after initial ATRA/ATO based therapy. New drug combinations and novel drug formulations continue to be developed with the goal of optimizing APL therapy, minimizing toxicities and making therapy more convenient for patients.

\section{References}

1. Douer D. The epidemiology of acute promyelocytic leukemia. Balliere's Best Pract Clin Hematol. 2003; 16:357-367.

2. Estey E, Thall P, Kantarjian H, et al. Association between increased body mass index and a diagnosis of acute promyelocytic leukemia in patients with acute myeloid leukemia. Leukemia. 1997; 11:1661-1664. [PubMed: 9324286]

3. Scaglioni PP, Pandolfi PP. The theory of APL revisited. Curr Top Microbiol Immunol. 2007; 313:85-100. [PubMed: 17217040]

4. Huang ME, Ye YC, Chen SR, et al. Use of all-trans retinoic acid in the treatment of acute promyelocytic leukemia. Blood. 1988; 72:567-572. [PubMed: 3165295]

5. Castaigne S, Chomienne C, Daniel MT, et al. All-trans retinoic acid as a differentiation therapy for acute promyelocytic leukemia. I. Clinical results Blood. 1990; 76:1704-1709.

6. Warrell RP Jr, Frankel SR, Miller WH Jr, et al. Differentiation therapy of acute promyelocytic leukemia with tretinoin (all-trans-retinoic acid). N Engl J Med. 1991; 324:1385-1393. [PubMed: 1850498]

7. Chen ZX, Xue YQ, Zhang R, et al. A clinical and experimental study on all-trans retinoic acidtreated acute promyelocytic leukemia patients. Blood. 1991; 78:1413-1419. [PubMed: 1884013]

8. Fenaux P, Castaigne S, Dombret H, et al. All-transretinoic acid followed by intensive chemotherapy gives a high complete remission rate and may prolong remissions in newly diagnosed acute promyelocytic leukemia: a pilot study on 26 cases. Blood. 1992; 80:2176-2181. [PubMed: 1421389]

9. Kanamaru A, Takemoto Y, Tanimoto M, et al. All-trans retinoic acid for the treatment of newly diagnosed acute promyelocytic leukemia. Japan Adult Leukemia Study Group. Blood. 1995; 85:1202-1206. [PubMed: 7858250]

10. Tallman MS, Andersen JW, Schiffer CA, et al. All-trans-retinoic acid in acute promyelocytic leukemia. N Engl J Med. 1997; 337:1021-1028. [PubMed: 9321529]

11. Tallman MS, Nabhan C, Feusner JH, Rowe JM. Acute promyelocytic leukemia: evolving therapeutic strategies. Blood. 2002; 99:759-767. [PubMed: 11806975] 
**12. Lo-Coco F, Avvisati G, Vignetti M, et al. Retinoic acid and arsenic trioxide for acute promyelocytic leukemia. N Engl J Med. 2013; 369:111-121. Phase III trial that randomized standard risk patients with APL to treatment with ATRA/chemotherapy vs. ATRA/ATO. Results showed non-inferiority of ATRA/ATO regimen compared to ATRA/chemotherapy. [PubMed: 23841729]

13. Fenaux $\mathrm{P}$, Chastang $\mathrm{C}$, Chevret $\mathrm{S}$, et al. A randomized comparison of all transretinoic acid (ATRA) followed by chemotherapy and ATRA plus chemotherapy and the role of maintenance therapy in newly diagnosed acute promyelocytic leukemia. The European APL Group. Blood. 1999; 94:1192-1200. [PubMed: 10438706]

14. Mandelli F, Diverio D, Avvisati G, et al. Molecular remission in PML/RAR alpha-positive acute promyelocytic leukemia by combined all-trans retinoic acid and idarubicin (AIDA) therapy. Gruppo Italiano-Malattie Ematologiche Maligne dell'Adulto and Associazione Italiana di Ematologia ed Oncologia Pediatrica Cooperative Groups. Blood. 1997; 90:1014-1021. [PubMed: 9242531]

15. Sanz MA, Martin G, Rayon C, et al. A modified AIDA protocol with anthracycline-based consolidation results in high antileukemic efficacy and reduced toxicity in newly diagnosed PML/ RARalpha-positive acute promyelocytic leukemia. PETHEMA group. Blood. 1999; 94:30153021. [PubMed: 10556184]

16. Fenaux P, Le Deley MC, Castaigne S, et al. Effect of all transretinoic acid in newly diagnosed acute promyelocytic leukemia. Results of a multicenter randomized trial. European APL 91 Group. Blood. 1993; 82:3241-3249. [PubMed: 8241496]

17. Ades L, Sanz MA, Chevret S, et al. Treatment of newly diagnosed acute promyelocytic leukemia (APL): a comparison of French-Belgian-Swiss and PETHEMA results. Blood. 2008; 111:10781084. [PubMed: 17975017]

18. Sanz MA, Lo Coco F, Martin G, et al. Definition of relapse risk and role of nonanthracycline drugs for consolidation in patients with acute promyelocytic leukemia: a joint study of the PETHEMA and GIMEMA cooperative groups. Blood. 2000; 96:1247-1253. [PubMed: 10942364]

19. Sanz MA, Martin G, Gonzalez M, et al. Risk-adapted treatment of acute promyelocytic leukemia with all-trans-retinoic acid and anthracycline monochemotherapy: a multicenter study by the PETHEMA group. Blood. 2004; 103:1237-1243. [PubMed: 14576047]

20. Ades L, Chevret S, Raffoux E, et al. Is cytarabine useful in the treatment of acute promyelocytic leukemia? Results of a randomized trial from the European Acute Promyelocytic Leukemia Group. J Clin Oncol. 2006; 24:5703-5710. [PubMed: 17116939]

21. Nasr R, Guillemin MC, Ferhi O, et al. Eradication of acute promyelocytic leukemia-initiating cells through PML-RARA degradation. Nat Med. 2008; 14:1333-1342. [PubMed: 19029980]

22. Niu C, Yan H, Yu T, et al. Studies on treatment of acute promyelocytic leukemia with arsenic trioxide: remission induction, follow-up, and molecular monitoring in 11 newly diagnosed and 47 relapsed acute promyelocytic leukemia patients. Blood. 1999; 94:3315-3324. [PubMed: 10552940]

23. Soignet SL, Frankel SR, Douer D, et al. United States multicenter study of arsenic trioxide in relapsed acute promyelocytic leukemia. J Clin Oncol. 2001; 19:3852-3860. [PubMed: 11559723]

24. Estey E, Garcia-Manero G, Ferrajoli A, et al. Use of all-trans retinoic acid plus arsenic trioxide as an alternative to chemotherapy in untreated acute promyelocytic leukemia. Blood. 2006; 107:3469-3473. [PubMed: 16373661]

**25. Iland HJ, Bradstock K, Supple SG, et al. All-trans-retinoic acid, idarubicin, and IV arsenic trioxide as initial therapy in acute promyelocytic leukemia (APML4). Blood. 2012; 120:15701580. Used a consolidation regimen of ATO/ATRA, eliminating chemotherapy from the consolidation phase and reported excellent response rates and OS. [PubMed: 22715121]

**26. Powell BL, Moser B, Stock W, et al. Arsenic trioxide improves event-free and overall survival for adults with acute promyelocytic leukemia: North American Leukemia Intergroup Study C9710. Blood. 2010; 116:3751-3757. Added ATO to the consolidation phase and reported improved outcomes, particularly in high risk APL patients. [PubMed: 20705755]

27. Ravandi F, Estey E, Jones D, et al. Effective treatment of acute promyelocytic leukemia with alltrans-retinoic acid, arsenic trioxide, and gemtuzumab ozogamicin. J Clin Oncol. 2009; 27:504510. [PubMed: 19075265] 
28. Shen ZX, Shi ZZ, Fang J, et al. All-trans retinoic acid/As2O3 combination yields a high quality remission and survival in newly diagnosed acute promyelocytic leukemia. Proc Natl Acad Sci U S A. 2004; 101:5328-5335. [PubMed: 15044693]

29. Iland, HJCM.; Seymour, J. The Australasian Leukaemia and lymphoma group APML4 trial update on APL treatment down under [abstract]. 6th International Symposium on Acute Promyelocytic Leukemia Abstract Book; 2013 Sept 29-Oct 2; Rome, Italy.

30. National Comprehensive Cancer Network. Acute Myeloid Leukemia version 2.2013. http:// www.nccn.org/professionals/physician_gls/pdf/aml.pdf

31. Sanz MA, Montesinos P, Rayon C, et al. Risk-adapted treatment of acute promyelocytic leukemia based on all-trans retinoic acid and anthracycline with addition of cytarabine in consolidation therapy for high-risk patients: further improvements in treatment outcome. Blood. 2010; 115:5137-5146. [PubMed: 20393132]

*32. Coutre, SOM.; Powell, B., et al. A phase III randomized trial (S0521) of maintenance versus observation for patients with previously untreated low/intermediate risk acute promyelocytic leukemia. 6th International Symposium on Acute Promyelocytic Leukemia Abstract Book; 2013 Sept 29-Oct 2; Phase III randomized trial that compared maintenance therapy to no maintenance therapy in standard risk patients and reported no differences in relapse rates or OS

33. de la Serna J, Montesinos P, Vellenga E, et al. Causes and prognostic factors of remission induction failure in patients with acute promyelocytic leukemia treated with all-trans retinoic acid and idarubicin. Blood. 2008; 111:3395-3402. [PubMed: 18195095]

34. Micol JBRE, Boissel N, et al. Do early events excluding patients with acute promyelocytic leukemia (APL) from trial enrollment modify treatment result evaluation? Real-life management of 100 patients referred to the University Hospital Saint-Louis between 2000 and 2010. Blood (ASH Annual Meeting Abstracts). 2010; 116:473.

35. Lehmann S, Ravn A, Carlsson L, et al. Continuing high early death rate in acute promyelocytic leukemia: a population-based report from the Swedish Adult Acute Leukemia Registry. Leukemia. 2011; 25:1128-1134. [PubMed: 21502956]

36. Altman JK, Rademaker A, Cull E, et al. Administration of ATRA to newly diagnosed patients with acute promyelocytic leukemia is delayed contributing to early hemorrhagic death. Leuk Res. 2013; 37:1004-1009. [PubMed: 23768930]

37. Rahmé R, Thomas X, Recher C, et al. Early Deaths (ED) in Acute Promyelocytic Leukemia (APL) in France: A Retrospective Multicenter Study in 355 Patients (pts). Blood (ASH annual Meeting Abstracts). 2012; 120:Abstract 890.

38. Tallman MS, Altman JK. How I treat acute promyelocytic leukemia. Blood. 2009; 114:5126-5135. [PubMed: 19797519]

39. Tallman, MS.; Brenner, B.; de Serna, JL., et al. Leuk Res; Meeting report Acute promyelocytic leukemia-associated coagulopathy; 21 January 2004; London, United Kingdom. 2005. p. 347-351.

40. Barbui T, Finazzi G, Falanga A. The impact of all-trans-retinoic acid on the coagulopathy of acute promyelocytic leukemia. Blood. 1998; 91:3093-3102. [PubMed: 9558362]

41. Kelaidi C, Ades L, Fenaux P. Treatment of acute promyelocytic leukemia with high white cell blood counts. Mediterr J Hematol Infect Dis. 2011; 3:e2011038. [PubMed: 22084652]

42. Frankel SR, Eardley A, Lauwers G, et al. The "retinoic acid syndrome" in acute promyelocytic leukemia. Ann Intern Med. 1992; 117:292-296. [PubMed: 1637024]

43. Kelaidi C, Chevret S, De Botton S, et al. Improved outcome of acute promyelocytic leukemia with high WBC counts over the last 15 years: the European APL Group experience. J Clin Oncol. 2009; 27:2668-2676. [PubMed: 19414681]

44. Vahdat L, Maslak P, Miller WH Jr, et al. Early mortality and the retinoic acid syndrome in acute promyelocytic leukemia: impact of leukocytosis, low-dose chemotherapy, PMN/RAR-alpha isoform, and CD13 expression in patients treated with all-trans retinoic acid. Blood. 1994; 84:3843-3849. [PubMed: 7949141]

45. de Botton S, Sanz MA, Chevret S, et al. Extramedullary relapse in acute promyelocytic leukemia treated with all-trans retinoic acid and chemotherapy. Leukemia. 2006; 20:35-41. [PubMed: 16307026] 
46. Sanz MA, Grimwade D, Tallman MS, et al. Management of acute promyelocytic leukemia: recommendations from an expert panel on behalf of the European Leukemia Net. Blood. 2009; 113:1875-1891. [PubMed: 18812465]

47. Breccia M, Carmosino I, Diverio D, et al. Early detection of meningeal localization in acute promyelocytic leukaemia patients with high presenting leucocyte count. Br J Haematol. 2003; 120:266-270. [PubMed: 12542484]

48. Lengfelder E, Hofmann WK, Nolte F. Management of elderly patients with acute promyelocytic leukemia: progress and problems. Ann Hematol. 2013; 92:1181-1188. [PubMed: 23694997]

49. Park JH, Qiao B, Panageas KS, et al. Early death rate in acute promyelocytic leukemia remains high despite all-trans retinoic acid. Blood. 2011; 118:1248-1254. [PubMed: 21653939]

50. Ades L, Chevret S, De Botton S, et al. Outcome of acute promyelocytic leukemia treated with all trans retinoic acid and chemotherapy in elderly patients: the European group experience. Leukemia. 2005; 19:230-233. [PubMed: 15565164]

51. Ono T, Takeshita A, Kishimoto Y, et al. Long-term outcome and prognostic factors of elderly patients with acute promyelocytic leukemia. Cancer Sci. 2012; 103:1974-1978. [PubMed: 22834728]

52. Sanz MA, Vellenga E, Rayon C, et al. All-trans retinoic acid and anthracycline monochemotherapy for the treatment of elderly patients with acute promyelocytic leukemia. Blood. 2004; 104:3490 3493. [PubMed: 15292063]

53. Latagliata R, Breccia M, Fazi P, et al. GIMEMA AIDA 0493 amended protocol for elderly patients with acute promyelocytic leukaemia. Long-term results and prognostic factors. Br J Haematol. 2011; 154:564-568. [PubMed: 21751984]

54. Zhang Y, Zhang Z, Li J, et al. Long-term efficacy and safety of arsenic trioxide for first-line treatment of elderly patients with newly diagnosed acute promyelocytic leukemia. Cancer. 2013; 119:115-125. [PubMed: 22930197]

55. Lo-Coco F, Cimino G, Breccia M, et al. Gemtuzumab ozogamicin (Mylotarg) as a single agent for molecularly relapsed acute promyelocytic leukemia. Blood. 2004; 104:1995-1999. [PubMed: 15187030]

56. Breccia M, Cimino G, Diverio D, et al. Sustained molecular remission after low dose gemtuzumabozogamicin in elderly patients with advanced acute promyelocytic leukemia. Haematologica. 2007; 92:1273-1274. [PubMed: 17768126]

57. Lo-Coco F, Orlando SM, Platzbecker U. Treatment of acute promyelocytic leukemia. N Engl J Med. 2013; 369:1472. [PubMed: 24106948]

58. Soignet SL, Maslak P, Wang ZG, et al. Complete remission after treatment of acute promyelocytic leukemia with arsenic trioxide. N Engl J Med. 1998; 339:1341-1348. [PubMed: 9801394]

59. Au WY, Lie AK, Chim CS, et al. Arsenic trioxide in comparison with chemotherapy and bone marrow transplantation for the treatment of relapsed acute promyelocytic leukaemia. Ann Oncol. 2003; 14:752-757. [PubMed: 12702530]

60. Raffoux E, Rousselot P, Poupon J, et al. Combined treatment with arsenic trioxide and all-transretinoic acid in patients with relapsed acute promyelocytic leukemia. J Clin Oncol. 2003; 21:23262334. [PubMed: 12805334]

61. Shigeno K, Naito K, Sahara N, et al. Arsenic trioxide therapy in relapsed or refractory Japanese patients with acute promyelocytic leukemia: updated outcomes of the phase II study and postremission therapies. Int J Hematol. 2005; 82:224-229. [PubMed: 16207595]

62. Aribi A, Kantarjian HM, Estey EH, et al. Combination therapy with arsenic trioxide, all-trans retinoic acid, and gemtuzumab ozogamicin in recurrent acute promyelocytic leukemia. Cancer. 2007; 109:1355-1359. [PubMed: 17326049]

63. Lu J, Huang X, Bao L, et al. Treatment outcomes in relapsed acute promyelocytic leukemia patients initially treated with all- retinoic acid and arsenic compound-based combined therapies. Oncol Lett. 2014; 7:177-182. [PubMed: 24348844]

64. Thirugnanam R, George B, Chendamarai E, et al. Comparison of clinical outcomes of patients with relapsed acute promyelocytic leukemia induced with arsenic trioxide and consolidated with either an autologous stem cell transplant or an arsenic trioxide-based regimen. Biol Blood Marrow Transplant. 2009; 15:1479-1484. [PubMed: 19822309] 
65. de Botton S, Fawaz A, Chevret S, et al. Autologous and allogeneic stem-cell transplantation as salvage treatment of acute promyelocytic leukemia initially treated with all-trans-retinoic acid: a retrospective analysis of the European acute promyelocytic leukemia group. J Clin Oncol. 2005; 23:120-126. [PubMed: 15534358]

66. Pemmaraju N, Tanaka MF, Ravandi F, et al. Outcomes in patients with relapsed or refractory acute promyelocytic leukemia treated with or without autologous or allogeneic hematopoietic stem cell transplantation. Clin Lymphoma Myeloma Leuk. 2013; 13:485-492. [PubMed: 23769669]

**67. Zhu HH, Wu DP, Jin J, et al. Oral tetra-arsenic tetra-sulfide formula versus intravenous arsenic trioxide as first-line treatment of acute promyelocytic leukemia: a multicenter randomized controlled trial. J Clin Oncol. 2013; 31:4215-4221. Randomized controlled trial comparing outcomes with IV arsenic vs. novel form of oral arsenic. Demonstrated no difference in outcomes with new formulation. [PubMed: 24127444]

68. Ozpolat B, Lopez-Berestein G, Adamson P, et al. Pharmacokinetics of intravenously administered liposomal all-trans-retinoic acid (ATRA) and orally administered ATRA in healthy volunteers. J Pharm Pharm Sci. 2003; 6:292-301. [PubMed: 12935441]

69. Tsimberidou AM, Tirado-Gomez M, Andreeff M, et al. Single-agent liposomal all-trans retinoic acid can cure some patients with untreated acute promyelocytic leukemia: an update of The University of Texas M. D. Anderson Cancer Center Series. Leuk Lymphoma. 2006; 47:10621068. [PubMed: 16840198]

70. Jain P, Kantarjian H, Estey E, et al. Single-Agent Liposomal All-Trans-Retinoic Acid as Initial Therapy for Acute Promyelocytic Leukemia: 13-Year Follow-Up Data. Clin Lymphoma Myeloma Leuk. 2013

71. Warrell RP Jr. Retinoid resistance in acute promyelocytic leukemia: new mechanisms, strategies, and implications. Blood. 1993; 82:1949-1953. [PubMed: 8400247]

72. Shao W, Benedetti L, Lamph WW, et al. A retinoid-resistant acute promyelocytic leukemia subclone expresses a dominant negative PML-RAR alpha mutation. Blood. 1997; 89:4282-4289. [PubMed: 9192750]

73. Ohnishi K. PML-RARalpha inhibitors (ATRA, tamibaroten, arsenic troxide) for acute promyelocytic leukemia. Int J Clin Oncol. 2007; 12:313-317. [PubMed: 17929112]

74. Tobita T, Takeshita A, Kitamura K, et al. Treatment with a new synthetic retinoid, Am80, of acute promyelocytic leukemia relapsed from complete remission induced by all-trans retinoic acid. Blood. 1997; 90:967-973. [PubMed: 9242525]

75. Naina HV, Levitt D, Vusirikala M, et al. Successful treatment of relapsed and refractory extramedullary acute promyelocytic leukemia with tamibarotene. J Clin Oncol. 2011; 29:e534536. [PubMed: 21482998]

76. Di Veroli A, Ramadan SM, Divona M, et al. Molecular remission in advanced acute promyelocytic leukaemia after treatment with the oral synthetic retinoid Tamibarotene. Br J Haematol. 2010; 151:99-101. [PubMed: 20618325] 


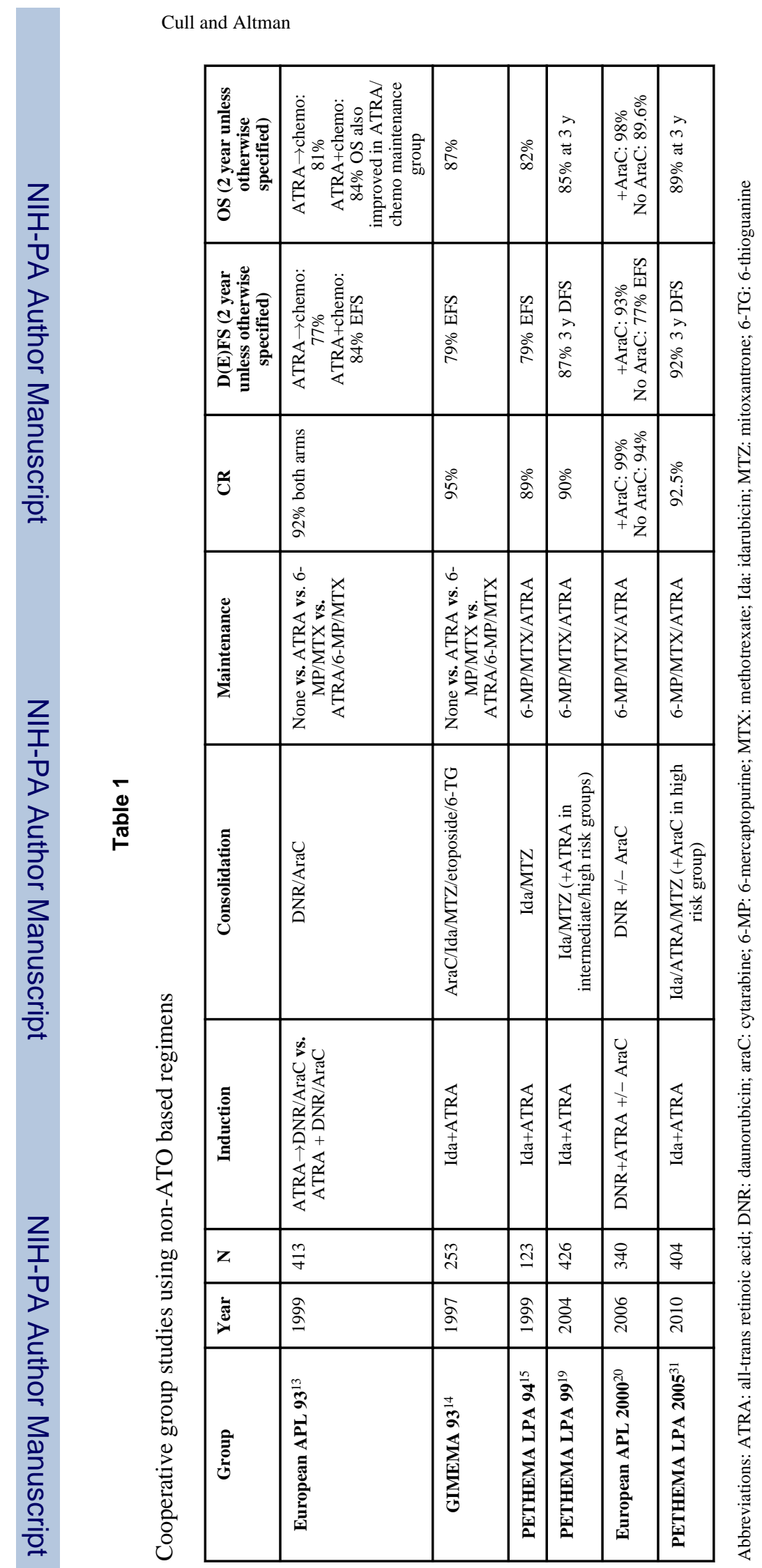

Curr Hematol Malig Rep. Author manuscript; available in PMC 2014 August 13. 
